J Pediatr Hematol Oncol. 2018 November ; 40(8): 574-579. doi:10.1097/MPH.0000000000001242.

\title{
Early Axial Growth Outcomes of Pediatric Patients Receiving Proton Craniospinal Irradiation
}

\author{
Brian De, BA ${ }^{1,2}$, Oren Cahlon, MD $^{1,2}$, Kevin Sine, BS $^{2}$, Dennis Mah, $\mathrm{PhD}^{2}$, Eugen B. Hug, \\ $\mathbf{M D}^{2}$, and Suzanne L. Wolden, MD ${ }^{1,2}$ \\ ${ }^{1}$ Department of Radiation Oncology, Memorial Sloan Kettering Cancer Center, 1275 York Avenue, \\ New York, NY 10065, USA \\ ${ }^{2}$ ProCure Proton Therapy Center, 103 Cedar Grove Lane, Somerset, NJ 08873, USA
}

\section{Abstract}

Guidelines on proton craniospinal irradiation (p-CSI) target volume selection in children are lacking. We examined the impact of target volume selection on growth of children receiving p-CSI at one institution. Records of 58 patients who received p-CSI were reviewed. Median age at treatment initiation was 8 years (range, 2-18 years). Spinal target volumes included whole vertebral body (WVB) in 67\% and partial vertebral body (PVB) in 33\%. Height z-scores before and after p-CSI were assessed using CDC stature-for-age charts. Maximal Cobb angle and height z-score change were compared for WVB vs. PVB p-CSI using a t-test. Among 93\% of patients with detailed data, median follow-up was 19 months (range, 2-58 months) after RT initiation. Quantitative growth evaluations were available for $64 \%$ of patients. Median change in height zscore was -0.5 (range, -2.1 to +0.7$)$ after treatment, representing a decrease $(\mathrm{p}<0.001)$ in ageadjusted height. WVB patients had significantly greater reduction in height z-score vs. PVB patients $(\mathrm{p}=0.004)$ but no difference in Cobb angle change $(\mathrm{p}>0.05)$. Despite reluctance surrounding its use in younger patients, PVB p-CSI was associated with similar spinal curvature and less growth suppression as compared to WVB p-CSI; a trial comparing WVB vs. PVB in children may be warranted.

\section{Keywords}

proton therapy; craniospinal irradiation; growth outcomes; pediatric oncology; central nervous system tumors

\section{Introduction}

Craniospinal irradiation (CSI) is an important component of therapy for children who are either at risk for or affected by disseminated disease throughout the neuraxis. However, use

Corresponding author: Suzanne L. Wolden, MD, Memorial Sloan Kettering Cancer Center, Department of Radiation Oncology, 1275 York Avenue, New York, NY 10065, Phone: 212-639-5148, Fax: 212-639-2417, woldens@ mskcc.org.

Abstract presented at the 59th Annual Meeting of the American Society for Radiation Oncology (ASTRO), San Diego, CA on September 24-27, 2017.

Conflicts of interest:

The authors have no conflicts to disclose. 
of CSI is associated with growth changes, particularly for younger patients. Radiation therapy (RT) to the vertebral body has been linked with the development of spinal curvature deformity. [1-3] In addition, while children treated with CSI can experience delayed-onset growth retardation secondary to endocrine abnormalities, spine-directed RT also leads to slowed vertebral body growth, leading to height deficits. [4-7] Due to dose reduction to anterior structures, proton CSI (p-CSI) offers a potential reduction of long-term sequelae over photon CSI, including cardiac dysfunction, hypothyroidism, second malignancy, and growth suppression. [8-12]

With current technology, two spinal target volume selection strategies are widely used for pCSI: whole vertebral body (WVB) and partial vertebral body (PVB). For children at skeletal maturity, only the thecal sac is intentionally targeted; however, the posterior portion of the vertebral body is included the planning target volume (PTV) to account for range uncertainties, leading to a functional PVB irradiation. For younger, growing children, PVB $\mathrm{p}$-CSI is generally discouraged since it is hypothesized to cause lordosis and scoliosis as a result of dose asymmetry across the vertebral body. $[1,13]$ To reduce the risk of lateral vertebral body wedging and spinal curvature deformity associated with lateral dose asymmetry for growing children [14, 15], the whole vertebral body (WVB) is targeted. Nonetheless, the use of WVB p-CSI may potentially undermine potential advantages of using p-CSI, including preservation of bone marrow, sparing of the anterior weight-bearing spine and organs at risk, and suppression of growth.

Choice of WVB vs. PVB in growing children receiving p-CSI to the whole spine remains controversial and practice patterns vary greatly. $[1,16]$ Owing to a paucity of published data, standardized guidelines on appropriate p-CSI target volume in children are lacking. We therefore report our early experience with dose selection, target volume selection, and early growth outcomes of children receiving p-CSI.

\section{Patients and Methods}

\section{Patient population}

This is a single-institution cohort of children treated with p-CSI. After approval by our internal Institutional Review Board, 58 patients aged 18 or younger at the time of treatment with p-CSI at our institution between 2012-2016 were identified. Gender composition was $69 \%$ male and $31 \%$ female. Median age at initiation of p-CSI treatment was 8 years (range, 2-18). Median age at treatment for males was 6 years (range, 2-14 years) for WVB and 15 years (range, 11-17 years) for PVB. For females, median age at treatment was 7 years (range, 6-11 years) for WVB and 13 years (range, 12-18 years) for PVB. The most common primary tumors were medulloblastoma (59\%), pineoblastoma (10\%), and neuroblastoma (9\%); a complete list of primary diagnoses and frequencies can be found in Table 1.

\section{Treatment planning and delivery}

Computed tomography(CT)-based simulation was performed for each patient. Volume delineation was conducted with axial CT images fused to diagnostic magnetic resonance images. Patients were positioned prone or supine for CSI and immobilized using an 
Aquaplast mold (Qfix Positioning, Avondale, PA) for the head and Alpha Cradle (Smithers Medical Products, North Canton, $\mathrm{OH}$ ) for the torso.

The craniospinal target volume consisted of the whole brain plus the length of the spinal canal to the level of the inferior aspect of the thecal sac. In cases where a tumor bed boost was used, the clinical target volume consisted of the tumor bed and areas of gross disease plus a margin, while respecting anatomic boundaries. Multiple beam approaches were used to treat the whole brain, including opposed laterals, posterior obliques, and a single posteroanterior (PA) portal. The portals ensured adequate margin on the cribriform plate. The cranial and spinal fields were matched with a daily feathering junction employing 3-4 match lines to spread out the hot spots and improve homogeneity in uniform scanning. While utilizing pencil beam scanning (PBS), a gradient structure allowing for a gradual match line was used in lieu of feathered match lines. $[17,18]$ The majority of spine volumes were treated using a single PA beam, while some cases utilized left and right posterior oblique portals to treat the spine volume. Spinal target volumes were chosen based on multiple factors, including patient age, gender, and remaining growth potential. Target volumes along the entire spinal axis included WVB in 67\% and PVB in 33\%. Normal structures, including but not limited to the pituitary gland, hypothalamus, brainstem, and spinal cord were contoured and dose constraints were determined as per QUANTEC/Emami guidelines. Daily orthogonal radiographs were used to ensure accurate setup and younger children were given daily anesthesia. Treatment was delivered using a rotational gantry.

Treatment with p-CSI was administered for primary treatment in $91 \%$ of patients and for CNS relapse for $9 \%$. As part of combined modality therapy, $79 \%$ received surgery and $12 \%$ received chemotherapy prior to p-CSI. Chemotherapy was administered concurrently with and/or after $\mathrm{p}$-CSI in $72 \%$. The $\mathrm{p}$-CSI dose was prescribed in radiobiological gray equivalents (Gy[RBE]) using the relative biologic effectiveness (RBE) value of 1.1, and dose was delivered in $1.8 \mathrm{~Gy}(\mathrm{RBE})$ fractions. Most common doses were 23.4, 36, and 18 Gy(RBE) used for $40 \%, 36 \%$, and $16 \%$ of patients, respectively. Boost RT to 1 or more sites was additionally given to all patients with cumulative median dose $54 \mathrm{~Gy}(\mathrm{RBE})$ [range, 18$59.4 \mathrm{~Gy}(\mathrm{RBE})]$. Boosted sites were supratentorial in $36 \%$, infratentorial in $67 \%$, and spinal in $14 \%$.

\section{Endpoints and statistical analysis}

The development of new-onset scoliosis was determined by physical examination at each follow-up visit and/or with imaging of the spine (MR spine, MR abdomen, CT scan, and/or chest x-ray). For 16 patients (28\%) for whom follow-up images were available, the maximum Cobb angle, defined as the maximum angle between lines extrapolated from rostral and caudal edges of a focus of scoliotic curvature, was measured. A Cobb angle of $>10^{\circ}$ was used to identify scoliosis. $[16,19]$ Cobb angles before and after treatment were compared using a t-test. Cobb angle change was additionally compared between patients receiving WVB and PVB p-CSI.

Changes in height were assessed by comparing patient height at baseline and during followup visits. Baseline height was obtained $<3$ months prior to p-CSI. A z-score, a statistical measure of the number of standard deviations away from the population mean that a 
particular data value lies, was calculated for baseline and the latest follow-up height using the 2000 Centers for Disease Control and Prevention growth charts. Subsequently, paired ttests were performed to compare changes in height z-scores before and after p-CSI. A twotailed t-test comparing z-score change in height between WVB and PVB p-CSI patients was also conducted. Linear regression with suppressed constant was used to assess change in height z-score vs. follow-up interval. Multivariate linear regression with suppressed constant adjusted for follow-up interval, sex, disease relapse status, p-CSI dose, target volume, and age at treatment was also performed.

Statistical analysis was performed with Microsoft Excel 2016 (Microsoft, Redmond, WA) and Stata Version 13.0 (StataCorp, College Station, TX). A p-value of $₫ \mathbf{0 . 0 5}$ using a 2-sided hypothesis was used to determine significance for all comparisons.

\section{Results}

Detailed follow-up data was available for 93\% of patients with a median follow-up of 19 months (range, 2-58 months) after RT initiation; data availability is displayed in Figure 1. Examination of the spine for curvature was conducted in $64 \%$ of patients with physical exam and imaging (59\% spine MRI, 3\% CT abdomen/pelvis, $2 \%$ chest $\mathrm{x}$-ray) and in $29 \%$ of patients as part of physical exam only. Among patients with radiographic evaluation, newonset MRI abnormalities included straightening of cervical lordosis (4\%) and mild midthoracic scoliotic curvature (2\%). No patients developed clinical or radiographic evidence of lordosis or scoliosis. In $28 \%$ of patients for whom maximum Cobb angles were calculated, the median was $2.7^{\circ}$ (range, $0.7^{\circ}-5.9^{\circ}$ ) before treatment and $3.8^{\circ}$ (range, $1.2^{\circ}-$ $9.4^{\circ}$ ) after treatment. A t-test of pre- and post-treatment yielded a significant increase in maximum Cobb angle $(\mathrm{p}<0.01)$. However, no patient met the scoliosis diagnostic criterion of a Cobb angle of $\geq 10^{\circ}$ at any time. Median Cobb angle change for patients receiving WVB was $+0.8^{\circ}$ (range $+0.2^{\circ}$ to $+7.2^{\circ}$ ) and for patients receiving PVB was $+1.4^{\circ}$ (range, $-0.8^{\circ}$ to $\left.+4.7^{\circ}\right)$; no difference was found on t-test $(\mathrm{p}=0.56)$.

Quantitative growth curve evaluations were available for 37 patients (64\%). At baseline, the median height percentile and z-score was $43.2 \%$ (range, $0.3-91.5 \%$ ) and -0.3 (range, -2.8 to +1.2 ). With median interval between height measurements of 24 months (range, 3-58 months), the median height percentile and z-score post-treatment was $24.4 \%$ (range, 0.3$85.8 \%$ ) and -0.8 (range, -2.9 to +1.1$)$, representing a decrease $(\mathrm{p}<0.001)$ in age-adjusted height compared to baseline. The median change in height $z$-score was -0.5 (range, -2.1 to +0.7 ). Z-score change after treatment was negative for 32 patients and positive for 5 patients. A comparison of height z-scores before and after treatment for WVB and PVB p-CSI are displayed in boxplot and connected scatterplot forms in Figure 2. Median change in height z-score among WVB patients was -0.6 (range, -2.1 to +0.7 ) and among PVB patients was -0.2 (range, -1.2 to +0.7 ); a significant difference between these groups was found on t-test $(\mathrm{p}=0.004)$.

Univariate linear regression analysis evaluating change in height z-score vs. follow-up interval is displayed in Figure 3, with resulting equation $\Delta z=-0.25 t$, where $\Delta z$ represents the change in height $\mathrm{z}$-score after p-CSI and $\mathrm{t}$ represents the follow-up interval between 
height measurements in years $\left(\mathrm{R}^{2}=0.16, \mathrm{p}<0.0001\right)$. On multivariate linear regression, significant negative contributions to change in height z-score were identified for follow-up interval and dose in Gy(RBE) and positive contribution was identified for age at p-CSI treatment $(\mathrm{p}<0.005)$. In the multivariate model, a year of follow-up after $\mathrm{p}$-CSI was associated with a -0.23 change in height $z$-score ( $95 \%$ confidence interval [CI], -0.37 to $-0.09)$. An increase in dose of $10 \mathrm{~Gy}(\mathrm{RBE})$ was associated with a height $\mathrm{z}$-score change of -0.34 (CI, -0.56 to -0.12 ). An incremental 5 years of age at diagnosis was associated with a height z-score change of $+0.43(\mathrm{CI},+0.20$ to +0.67$)$. Gender, relapse status, use of concurrent/adjuvant chemotherapy, and target volume were not associated with change in height z-score. Regression results are displayed in Table 2.

\section{Discussion}

Dose and target volume selection in p-CSI for pediatric patients may be guided by disease characteristics, comorbidities, age, gender, and remaining growth potential, with the goal of controlling disease while minimizing deformity. While several studies have advocated for including the WVB in patients under 15 years of age [20-22], approximating the age of skeletal maturity, the approach is ultimately physician- and patient-dependent. In our study, all patients aged $<10$ years received WVB $p$-CSI, whereas those aged $>14$ years received PVB p-CSI. Target volumes for patients aged 10-14 years were given more careful consideration and chosen based on patient characteristics and preferences elicited after informed consent with patients' families.

The risk of post-treatment scoliosis is the most common reason for including the WVB in the target volume for p-CSI in growing children, but it is unclear if WVB p-CSI mitigates this risk. Potential risk factors for scoliosis with RT to any part of the spine include dose $>24$ Gy and larger field size. [3,16] Previous literature has documented late effects of photon RT to the spine for Wilms tumor, neuroblastoma, and medulloblastoma, with long-term rates ranging between 40-50\% for pediatric patients despite the inclusion of the WVB in the target volume. [1] Among patients treated with PVB p-CSI to the entire spine, one study by MacEwan, et al. with a median follow-up of over 13 years reported that 2 of 5 pediatric medulloblastoma patients developed scoliosis. While we report a statistically significant increase in Cobb angle after treatment, we did not find any instances of clinically- or radiographically-diagnosed scoliosis at a median follow-up of approximately 1 year, reaffirming that scoliosis is not a common early effect of p-CSI. However, we also did not find any difference in Cobb angle change between patients receiving WVB vs. PVB p-CSI, suggesting that PVB p-CSI may not be associated with greater development of scoliosis.

Our study demonstrates that the effects on age-adjusted height may be seen shortly after pCSI treatment. Time to follow-up was significantly associated with a decrease in height $\mathrm{z}$ score, both on univariate and multivariate analysis, reflecting the impact of both target volume strategies on epiphyseal growth plates. Age at treatment and RT dose were also associated with change in height z-score, as has been shown for photon CSI in previous studies. [2, 5, 6, 23] However, other attributes such as gender, relapse status, chemotherapy, and target volume were not associated with change in height $\mathrm{z}$-score. These results suggest that the effect of RT on the ossification centers of the vertebrae may more profoundly 
suppress growth than other factors in the immediate post-RT period. Our findings regarding PVB p-CSI suggest that it is a potentially superior target volume strategy even for younger patients, since its effects on growth suppression and lateral spinal curvature deformity may be non-inferior to those for WVB p-CSI. Though longer follow-up with this cohort of patients is needed to confirm these findings, the potential benefits of PVB p-CSI include reduction of both short- and long-term toxicity and secondary malignancy. [9] While there has been reluctance to pursue PVB p-CSI given the concern of scoliosis, our data show that it may be feasible and safe in children; a clinical trial evaluating PVB p-CSI against WVB pCSI in younger children is warranted. A trial evaluating pCSI with bone sparing is underway (NCT03281889) [24] but a direct head-to-head comparison of WVB vs. PVB p-CSI may better quantify the growth benefits of bone sparing.

The development of scoliosis and vertical growth suppression are multifactorial, and other etiologies for these growth abnormalities must be acknowledged. Burden of systemic disease, dose and duration of steroids used, chemotherapy, and hormonal dysfunction after RT may contribute to growth suppression. [25, 26] Cerebellar damage related to tumor or resection in posterior fossa tumors, vascular injury secondary to treatment, and uneven growth of paravertebral/paraspinal muscles may contribute to the development of scoliosis. While these effects were not captured in the present study, many of them would be unlikely to manifest in the early post-RT time frame examined.

Both target volume strategies discussed have drawbacks. At our institution, WVB treatment allows a margin of $0.3-0.5 \mathrm{~cm}$ from the anterior aspect of the vertebral body to avoid dose delivery to anterior structures, resulting in non-uniform posterior-to-anterior dose exposure. While no instances of lordosis were found to date, continued follow up is needed to ascertain the longer-term implications of this margin. PVB p-CSI spares the majority of the vertebral body, but some dose is ultimately delivered to the posterior aspect of the vertebral body, possibly including one or more ossification centers in growing bone. [13] While PVB p-CSI may be effective in reducing bone marrow toxicity and/or lateral curvature deformity, it remains unclear if PVB p-CSI mitigates growth inhibition in spite of this incidentally delivered dose. This effect may be compounded by possibility of increased RBE a few millimeters beyond the Bragg peak, known as the distal fall-off. [27] Our study found a significantly reduced growth suppression on univariate analysis but this was not reproduced on multivariate analysis. Other institutions have also reported use of alternate partial vertebral body irradiation strategies [1], conformal thecal sac-only p-CSI using intensitymodulated proton therapy (IMPT), and PBS that spares ossification centers [28, 29]. In addition, novel imaging techniques such as dual energy $\mathrm{CT}$ and prompt gamma imaging may reduce range uncertainty and permit more precise dose delivery. [30, 31] Future studies should explore the effects of these tools, which may enable greater vertebral body sparing and reduced growth inhibition compared to conventional methods.

Limitations of our study include the small sample size, retrospective design, heterogeneous patient population and management, substantial loss to quantitative follow up, and probable confounders. However, to the best of our knowledge, this study is one of the first to use a large cohort to quantitatively estimate the early effects of p-CSI on growth changes, including spinal curvature deformity and age-adjusted height suppression. Further 
investigation into novel strategies to avoid bone exposure and reduce range uncertainty is warranted. In the absence of more conformal techniques used in clinical practice, however, our results suggest that a prospective trial evaluating PVB vs. WVB p-CSI should be conducted to compare growth suppression, scoliosis, and short- and long-term toxicity in children.

\title{
Acknowledgments
}

\author{
Source of Funding
}

Funding was provided by the National Institutes of Health/National Cancer Institute Cancer Center Support Grant (P30 CA008748). This work is also supported by a gift from Jack and Susan Rudin.

\section{References}

1. McMullen K, Buchsbaum J, Douglas J, McDonald M, Johnstone P. Growth abnormalities of the spine after radiation therapy: Respecting the past while moving forward in proton craniospinal irradiation. Practical radiation oncology. 2013 Oct-Dec;3(4):337-43. Epub 2014/03/29. eng. [PubMed: 24674407]

2. Hartley KA, Li C, Laningham FH, Krasin MJ, Xiong X, Merchant TE. Vertebral body growth after craniospinal irradiation. International journal of radiation oncology, biology, physics. 2008 Apr 01; 70(5):1343-9. Epub 2008/01/01. eng.

3. Neuhauser EB, Wittenborg MH, Berman CZ, Cohen J. Irradiation effects of roentgen therapy on the growing spine. Radiology. 1952 Nov; 59(5):637-50. Epub 1952/11/01. engund. [PubMed: 12994000]

4. Gawade PL, Hudson MM, Kaste SC, Neglia JP, Wasilewski-Masker K, Constine LS, et al. A systematic review of selected musculoskeletal late effects in survivors of childhood cancer. Current pediatric reviews. 2014; 10(4):249-62. Epub 2014/11/19. eng. [PubMed: 25403639]

5. Probert JC, Parker BR. The effects of radiation therapy on bone growth. Radiology. 1975 Jan; 114(1):155-62. Epub 1975/01/01. eng. [PubMed: 813276]

6. Shalet SM, Gibson B, Swindell R, Pearson D. Effect of spinal irradiation on growth. Archives of disease in childhood. 1987 May; 62(5):461-4. Epub 1987/05/01. eng. [PubMed: 3606177]

7. Hua C, Shukla HI, Merchant TE, Krasin MJ. Estimating differences in volumetric flat bone growth in pediatric patients by radiation treatment method. International journal of radiation oncology, biology, physics. 2007 Feb 01; 67(2):552-8. Epub 2006/11/14. eng.

8. Chhabra A, Mahajan A. Treatment of common pediatric CNS malignancies with proton therapy. Chinese clinical oncology. 2016 Aug.5(4):49. Epub 2016/08/11. eng. [PubMed: 27506805]

9. Brown AP, Barney CL, Grosshans DR, McAleer MF, de Groot JF, Puduvalli VK, et al. Proton beam craniospinal irradiation reduces acute toxicity for adults with medulloblastoma. International journal of radiation oncology, biology, physics. 2013 Jun 01; 86(2):277-84. Epub 2013/02/26. eng.

10. Yock TI, Yeap BY, Ebb DH, Weyman E, Eaton BR, Sherry NA, et al. Long-term toxic effects of proton radiotherapy for paediatric medulloblastoma: a phase 2 single-arm study. The Lancet Oncology. 2016 Mar; 17(3):287-98. Epub 2016/02/03. eng. [PubMed: 26830377]

11. Mahajan A. Proton Craniospinal Radiation Therapy: Rationale and Clinical Evidence. International Journal of Particle Therapy. 2014; 1(2):399-407.

12. Fossati P, Ricardi U, Orecchia R. Pediatric medulloblastoma: toxicity of current treatment and potential role of protontherapy. Cancer treatment reviews. 2009 Feb; 35(1):79-96. Epub 2008/11/04. eng. [PubMed: 18976866]

13. Paganetti H. Range uncertainties in proton therapy and the role of Monte Carlo simulations. Physics in medicine and biology. 2012 Jun 07; 57(11):R99-117. Epub 2012/05/11. eng. [PubMed: 22571913] 
14. Arkin AM, Simon N. Radiation scoliosis; an experimental study. The Journal of bone and joint surgery American volume. 1950 Apr; 32a(2):396-401. Epub 1950/04/01. eng. [PubMed: 15412181]

15. Arkin AM, Pack GT, Ransohoff NS, Simon N. Radiation-induced scoliosis; a case report. The Journal of bone and joint surgery American volume. 1950 Apr; 32a(2):401-4. Epub 1950/04/01. eng. [PubMed: 15412182]

16. MacEwan I, Chou B, Moretz J, Loredo L, Bush D, Slater JD. Effects of Vertebral Body Sparing Proton Craniospinal Irradiation on the Spine of Young Pediatric Patients with Medulloblastoma. Advances in Radiation Oncology. 2017; 2:220-7. [PubMed: 28740935]

17. Stoker JB, Grant J, Zhu XR, Pidikiti R, Mahajan A, Grosshans DR. Intensity modulated proton therapy for craniospinal irradiation: organ-at-risk exposure and a low-gradient junctioning technique. International journal of radiation oncology, biology, physics. 2014 Nov 01; 90(3):63744. Epub 2014/09/10. eng.

18. Lin H, Ding X, Kirk M, Liu H, Zhai H, Hill-Kayser CE, et al. Supine craniospinal irradiation using a proton pencil beam scanning technique without match line changes for field junctions. International journal of radiation oncology, biology, physics. 2014 Sep 01; 90(1):71-8. Epub 2014/07/17. eng.

19. Malfair D, Flemming AK, Dvorak MF, Munk PL, Vertinsky AT, Heran MK, et al. Radiographic evaluation of scoliosis: review. AJR American journal of roentgenology. 2010 Mar; 194(3 Suppl):S8-22. Epub 2010/03/05. eng. [PubMed: 20173177]

20. Merchant TE. Clinical Controversies: Proton Therapy for Pediatric Tumors. Seminars in Radiation Oncology. 2013; 23(2):97-108. [PubMed: 23473686]

21. Giebeler A, Newhauser WD, Amos RA, Mahajan A, Homann K, Howell RM. Standardized treatment planning methodology for passively scattered proton craniospinal irradiation. Radiation Oncology. 2013; 8(1):32. [PubMed: 23375151]

22. Howell RM, Giebeler A, Koontz-Raisig W, Mahajan A, Etzel CJ, D’Amelio AM Jr, et al. Comparison of therapeutic dosimetric data from passively scattered proton and photon craniospinal irradiations for medulloblastoma. Radiat Oncol. 2012 Jul 24.7:116. Epub 2012/07/26. eng. [PubMed: 22828073]

23. Xu W, Janss A, Moshang T. Adult height and adult sitting height in childhood medulloblastoma survivors. The Journal of clinical endocrinology and metabolism. 2003 Oct; 88(10):4677-81. Epub 2003/10/15. eng. [PubMed: 14557440]

24. Proton Craniospinal Irradiation With Bone Sparing to Decrease Growth Decrement From Radiation. https://ClinicalTrials.gov/show/NCT03281889

25. Chemaitilly W, Hudson MM. Update on endocrine and metabolic therapy-related late effects observed in survivors of childhood neoplasia. Current opinion in endocrinology, diabetes, and obesity. 2014 Feb; 21(1):71-6. Epub 2013/11/28. eng.

26. Christopherson KM, Rotondo RL, Bradley JA, Pincus DW, Wynn TT, Fort JA, et al. Late toxicity following craniospinal radiation for early-stage medulloblastoma. Acta Oncol. 2014 Apr; 53(4): 471-80. Epub 2014/02/26. eng. [PubMed: 24564687]

27. Krejcarek SC, Grant PE, Henson JW, Tarbell NJ, Yock TI. Physiologic and Radiographic Evidence of the Distal Edge of the Proton Beam in Craniospinal Irradiation. International journal of radiation oncology, biology, physics. 2007 Jul 1; 68(3):646-9. eng.

28. Giantsoudi D, Seco J, Eaton BR, Simeone FJ, Kooy H, Yock TI, et al. Evaluating Intensity Modulated Proton Therapy Relative to Passive Scattering Proton Therapy for Increased Vertebral Column Sparing in Craniospinal Irradiation in Growing Pediatric Patients. International journal of radiation oncology, biology, physics. 2017 May 1; 98(1):37-46. Epub 2017/06/08. eng.

29. Jiang S, Wang J, Li H, Liao L, Li Y, Wang X, et al. Novel Hybrid Scattering- and Scanning-Beam Proton Therapy Approach. International Journal of Particle Therapy. 2016; 3(1):37-50.

30. Yang M, Virshup G, Clayton J, Zhu XR, Mohan R, Dong L. Theoretical variance analysis of single- and dual-energy computed tomography methods for calculating proton stopping power ratios of biological tissues. Physics in medicine and biology. 2010 Mar 07; 55(5):1343-62. Epub 2010/02/11. eng. [PubMed: 20145291] 
31. Priegnitz M, Barczyk S, Nenoff L, Golnik C, Keitz I, Werner T, et al. Towards clinical application: prompt gamma imaging of passively scattered proton fields with a knife-edge slit camera. Physics in medicine and biology. 2016 Nov 21; 61(22):7881-905. Epub 2016/10/27. eng. [PubMed: 27779120] 


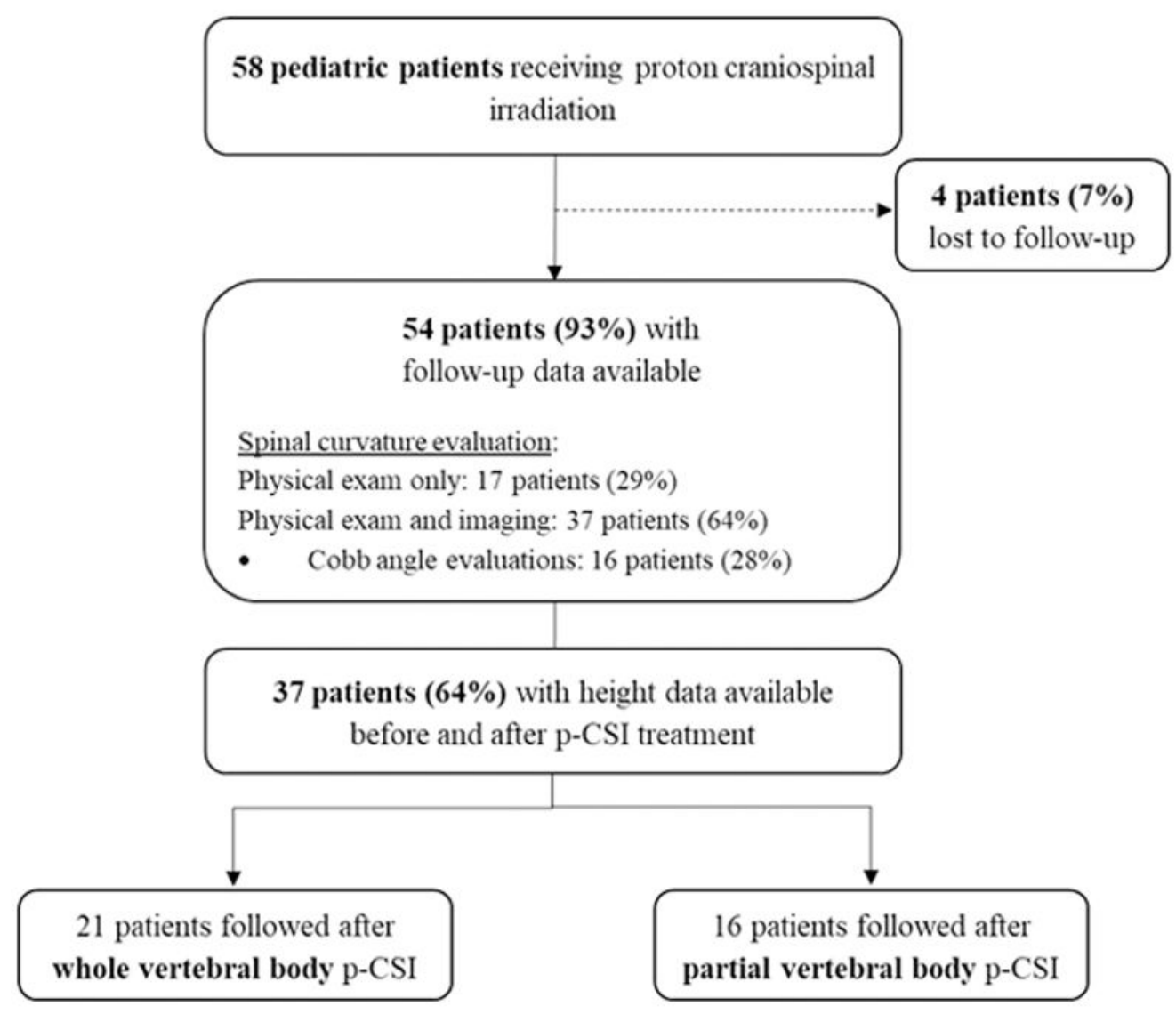

Figure 1.

CONSORT diagram of patients included in study. 

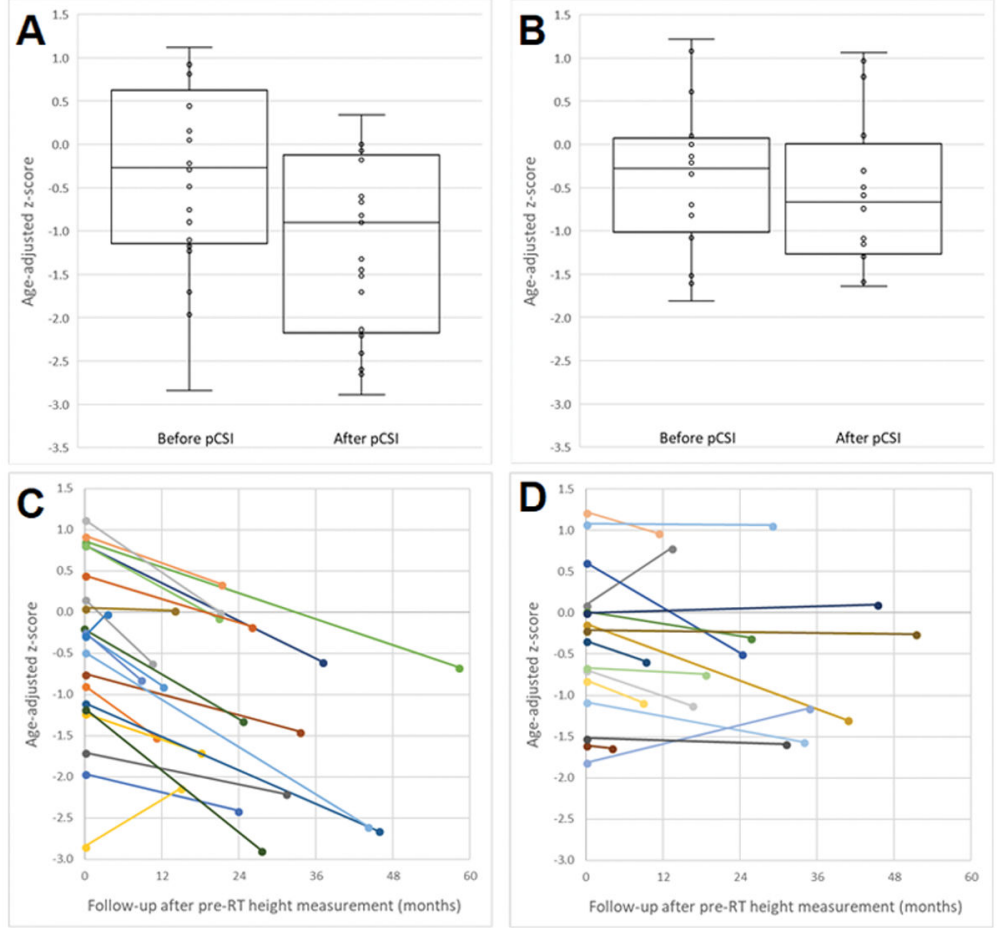

Figure 2.

Boxplots of height z-scores before and after (A) whole vertebral body p-CSI and (B) partial vertebral body $\mathrm{p}$-CSI and connected scatterplots of height $\mathrm{z}$-scores before and after $(\mathrm{C})$ whole vertebral body p-CSI and (D) partial vertebral body p-CSI. Median change in height Z-score among WVB patients was -0.6 (range, -2.1 to +0.7 ) and among PVB patients was -0.2 (range, -1.2 to +0.7 ); a significant difference between these groups was found on t-test $(\mathrm{p}=0.004)$. 


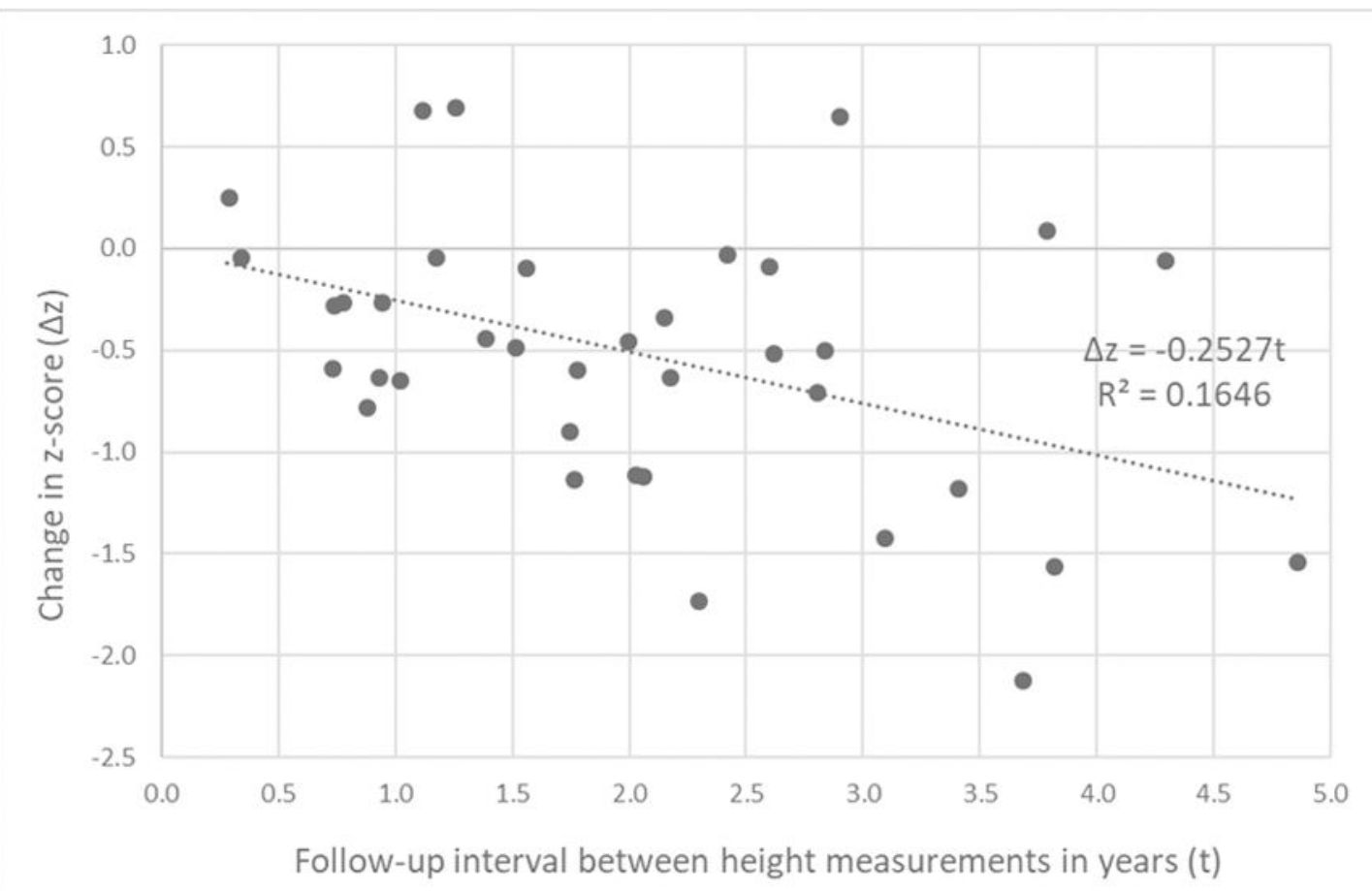

Figure 3.

Univariate linear regression of change in height $\mathrm{z}$-score after $\mathrm{p}$-CSI vs. interval between height measurements. $\left(\mathrm{R}^{2}=0.16, \mathrm{p}<0.0001\right)$ 


\section{Table 1}

List of primary diagnoses and frequencies of included patients receiving p-CSI

\begin{tabular}{l|l}
\hline Primary diagnosis & $\mathbf{n}(\%)$ \\
\hline Medulloblastoma & $34(59 \%)$ \\
\hline Pineoblastoma & $6(10 \%)$ \\
\hline Neuroblastoma & $5(9 \%)$ \\
\hline Germinoma & $3(5 \%)$ \\
\hline Non-germinoma germ cell tumor & $3(5 \%)$ \\
\hline Supratentorial PNET & $3(5 \%)$ \\
\hline Ependymoma & $1(2 \%)$ \\
\hline Oligodendroglioma & $1(2 \%)$ \\
\hline Choroid plexus carcinoma & $1(2 \%)$ \\
\hline Acute lymphoblastic leukemia & $1(2 \%)$ \\
\hline
\end{tabular}




\section{Table 2}

Results of multivariate linear regression of change in height z-score after p-CSI vs. interval between height measurements.

\begin{tabular}{l|l|l|l}
\hline Variable $^{*}$ & Coefficient & $\mathbf{9 5 \%}$ CI & p-value \\
\hline Follow-up interval (years) & $\mathbf{- 0 . 2 3 0}$ & $\mathbf{- 0 . 3 7 3}$ to $-\mathbf{0 . 0 8 7}$ & $\mathbf{0 . 0 0 3}$ \\
\hline Gender & -0.200 & -0.572 to +0.171 & 0.280 \\
\hline Relapse status & -0.008 & -0.531 to +0.515 & 0.975 \\
\hline Chemotherapy use & -0.052 & -0.429 to +0.326 & 0.782 \\
\hline Target volume & +0.127 & -0.356 to +0.610 & 0.596 \\
\hline Age at p-CSI & $\mathbf{+ 0 . 0 8 6}$ & $\mathbf{+ 0 . 0 3 9}$ to $+\mathbf{0 . 1 3 3}$ & $\mathbf{0 . 0 0 1}$ \\
\hline Dose in Gy(RBE) & $\mathbf{- 0 . 0 3 4}$ & $\mathbf{- 0 . 0 5 6}$ to $-\mathbf{0 . 0 1 2}$ & $\mathbf{0 . 0 0 4}$ \\
\hline
\end{tabular}

* Sex coded as $0=$ male, 1 female. Relapse status coded as $0=$ not relapsed, $1=$ relapsed. Chemotherapy coded as $0=$ no concurrent/adjuvant chemotherapy, $1=$ concurrent/adjuvant chemotherapy used. Target volume coded as $0=$ partial vertebral body, $1=$ whole vertebral body.

$\mathrm{CI}=$ confidence interval. $\mathrm{R}^{2}=0.74, \mathrm{p}<0.0001$ 